\title{
Seasonal abundance of Anopheles mosquitoes and their association with meteorological factors and malaria incidence in Bangladesh
}

\author{
Kabirul Bashar ${ }^{1 *}$ and Nobuko Tuno ${ }^{2}$
}

\begin{abstract}
Background: The relationship between climatic factors and mosquito abundance is very important to determine parasite activity levels and, therefore, disease risk. Therefore, this study was conducted to investigate the seasonal abundance of anophelines and their association with meteorological variables and disease transmission in two malaria endemic areas of Bangladesh.

Methods: Monthly sampling was done from both indoors and outdoors in 12 selected houses using light traps (LTs) and pyrethrum spray (PS) during January, 2011 to January, 2012 in two malaria endemic areas of Bangladesh. Outdoor rainfall, temperature, and relative humidity data of the study areas were collected from the meteorological department of Bangladesh. Mosquitoes were killed with chloroform and identified morphologically under stereoscopic microscopes using taxonomic keys. Samples were tested for CSP of P. falciparum, P. vivax 210 and P. vivax 247 using ELISA. Pearson correlation and canonical correspondence analyses (CCA) were computed to investigate the associations with species abundance and rainfall, temperature, humidity and malaria cases.
\end{abstract}

Results: A total of 2,443 female anophelines, representing 22 species were captured. Every female Anopheles were tested for $P$. falciparum, P. vivax 210 and P. vivax 247 CSP, of which 10 species were found positive. The CSP positive species were An. annularis, An. baimaii, An. barbirostris, An. jeyporiensis, An. karwari, An. minimus s.l., An. philippinensis, An. umbrosus, An. vagus and An. wilmori. Anopheles vagus and An. philippinensis were the dominant species present almost throughout the year with highest peaks in March and smallest peaks in September but An. baimaii and An. willmori were found during monsoon (July -September) only. Lag rainfall and relative humidity were the most significant variables influencing An. baimaii, An. willmori, An. vagus, and An. subpictus density in Kumari area. Abundance of these four species positively related to malaria cases. The effects of temperature were not found as a significant variable on the abundance of anophelines mosquitoes in Bangladesh.

Conclusions: Our study demonstrates that the nature of relationship between malaria vector and climatic variables were multifaceted. Detailed studies of vector bionomics, continuous monitoring and malaria transmission dynamics is essential for predicting disease outbreaks and vector control in the region.

\section{Background}

Malaria is one of the most formidable and serious public health problems in Bangladesh [1]. It is endemic in 13 northern and eastern areas bordering India and Myanmar, with $90 \%$ of morbidity and mortality reported from Rangamati, Bandarban and Khagrachari districts [2]. The malaria situation in Bangladesh is complex due to

\footnotetext{
* Correspondence: bkabirul@gmail.com

'Laboratory of Entomology, Department of Zoology, Jahangirnagar

University, Savar, Dhaka 1342, Bangladesh

Full list of author information is available at the end of the article
}

high species diversity and species complexes with many sibling species presenting different ecological behaviors [2-7]. Anopheles baimaii Sallum and Peyton, 2005 (dirus D), An. minimus s.l. Theobald, 2001, An. philippinensis Ludlow, 1902, and An. sundaicus (Rodenwaldt, 1925) are considered as primary and An. aconitus Doenitz, 1902, An. annularis Van der Wulp, 1884, and An. vagus Doenitz, 1902 as epidemic malaria vectors in Bangladesh [4]. However, recent studies $[1,8,9]$ have reported $A n$. nigerrimus Giles, 1900, An. subpictus Grassi, 1899, An. barbirostris Van der Wulp, 1884, An. maculates Theobald, 1901, An. 
jeyporiensis James,1902, An. karwari James, 1902, An. kochi Doenitz,1901, and An. peditaeniatus (Leicester, 1908) as Plasmodium positive.

Environmental changes have a great bearing on breeding habitats of different mosquito species that control adult population density [10]. Meteorological factors affect adult mosquito abundance by altering the quality and quantity of breeding habitats. The relationship between climate variables and mosquito abundance can provide important information to determine parasite activity levels and, therefore, disease risk [11-16]. Exact information on the seasonal prevalence of mosquito fauna in a region is essential for the development of efficient vector control programs [17]. But there were few published data on the seasonal abundance of anopheline mosquitoes in Bangladesh. Therefore, this study was conducted to investigate the species composition and population dynamics of anophelines and their association with meteorological variables and disease transmission in two malaria endemic areas of Bangladesh.

\section{Methods \\ Study areas}

Two malaria endemic areas of Bangladesh were selected for the study these are Kumari of Bandarban district $\left(21^{\circ} 44^{\prime} \mathrm{N}, 92^{\circ} 8^{\prime} \mathrm{E}\right)$ and Sreemangal of Maulvibazar district $\left(24^{\circ} 19^{\prime} \mathrm{N}, 91^{\circ} 46^{\prime} \mathrm{E}\right.$ ) (Figure 1). These areas were selected on the basis of the malaria prevalence and bio- geographical condition of Bangladesh. Infection rate in Kumari and Sreemangal was $5>$ and $0.001-1$ respectively [8]. A major part of Kumari is vegetated by secondary forest with interspersed rubber plantations. Hot and humid climatic conditions, narrow slow-running streams, wells, pools and rice fields in the area are the suitable breeding habitats for mosquitoes. Semi-evergreen coniferous forests are the major vegetation in Sreemangal. There are few slow running streams which acted as mosquito breeding sources. Ggeographical positions of the sampling places were recorded using handheld GPS (Garmin Oregon 550). ArcView GIS 3.3 and Arc GIS 9.2 software were used to map the sampling area.

\section{Mosquito collections and identification}

Mosquitoes sampling was done twice in a month during January to December' 2011 in Kumari and once in a month during February to December' 2011 and January' 2012 in Sreemangal. Mosquitoes were captured from both indoors and outdoors from 12 selected houses from the study areas using light traps (LTs) and pyrethrum spray (PS) following World Health Organization procedures [18]. Collected mosquitoes were brought to the field laboratory for processing and identification. Mosquitoes were killed with chloroform and identified morphologically under stereoscopic microscopes within 12 hour using taxonomic keys [19-22] and stored in Eppendorf tubes with soft tissue paper and silica gel desiccant for stable storage at room temperature for further process. Collected mosquitoes were transported to the laboratory at Jahangirnagar University to be preserved in freezer. The anophelines were brought to Kanazawa University later to confirm species identification.

\section{Sample preparation and CSP ELISA}

Each mosquito was bisected into head-thorax and abdomen. The anterior part, head, and thorax of the mosquitoes were tested. Samples were tested for CSP of Plasmodium falciparum (Pf), P. vivax (Pv) 210 and P. vivax 247 using ELISA as described by Burkot et al. [23] and Wirtz et al. [24] with slight modification. All monoclonal antibodies were collected from the Centers for Disease Control and Prevention (CDC), Atlanta. These were used to detect and distinguish the circumsporozoite proteins (CSP) of P. falciparum and two distinct polymorphs of $P$. vivax: $\mathrm{Pv}-210$ and $\mathrm{Pv}-247$. Four negative controls (each the triturated whole body of a laboratory reared, Aedes aegypti) and one positive control (supplied by CDC) were included in each microtitre plate. ELISA results (i.e. change in color) were visually judged according to the color intensity (no color change, negative result), comparable with the positive control. All positive samples were re-tested for confirmation. We considered the sample as CSP-positive, if two of two trials were positive. Recently Durnez et al. [25] and Bashar et al. [9] describe a new methodology to check the true CSP positive. They proved that, ELISA reacting antigen of Plasmodium was heat-stable but false positive causal agent was not. Therefore we confirm positive results by a second CSP-ELISA test applying heated the samples at $100^{\circ} \mathrm{C}$ for 10 minutes.

\section{Ethics statement}

Ethical clearance was obtained from the head of each village, office of the local government and the ethics committee of Bangladesh Medical Research Council (No. PU/203/11). We took verbal consent from all of the household head for this study because the majority of them are illiterate. The ethics committee approved this consent procedure.

\section{Malaria cases}

Data on the malaria cases ( $P f$ and $P v)$ of the study areas were received from the office of malaria control program and local Hospitals. Local hospitals routinely checked the $P$. falsiparum cases using rapid diagnostic test (RDT) and $P$. vivax cases by using thin and thick blood films.

\section{Meteorological data}

Outdoor rainfall, temperature and relative humidity data of the study areas, from January' 2011 to February' 2012 were collected from the local stations of the meteorological 


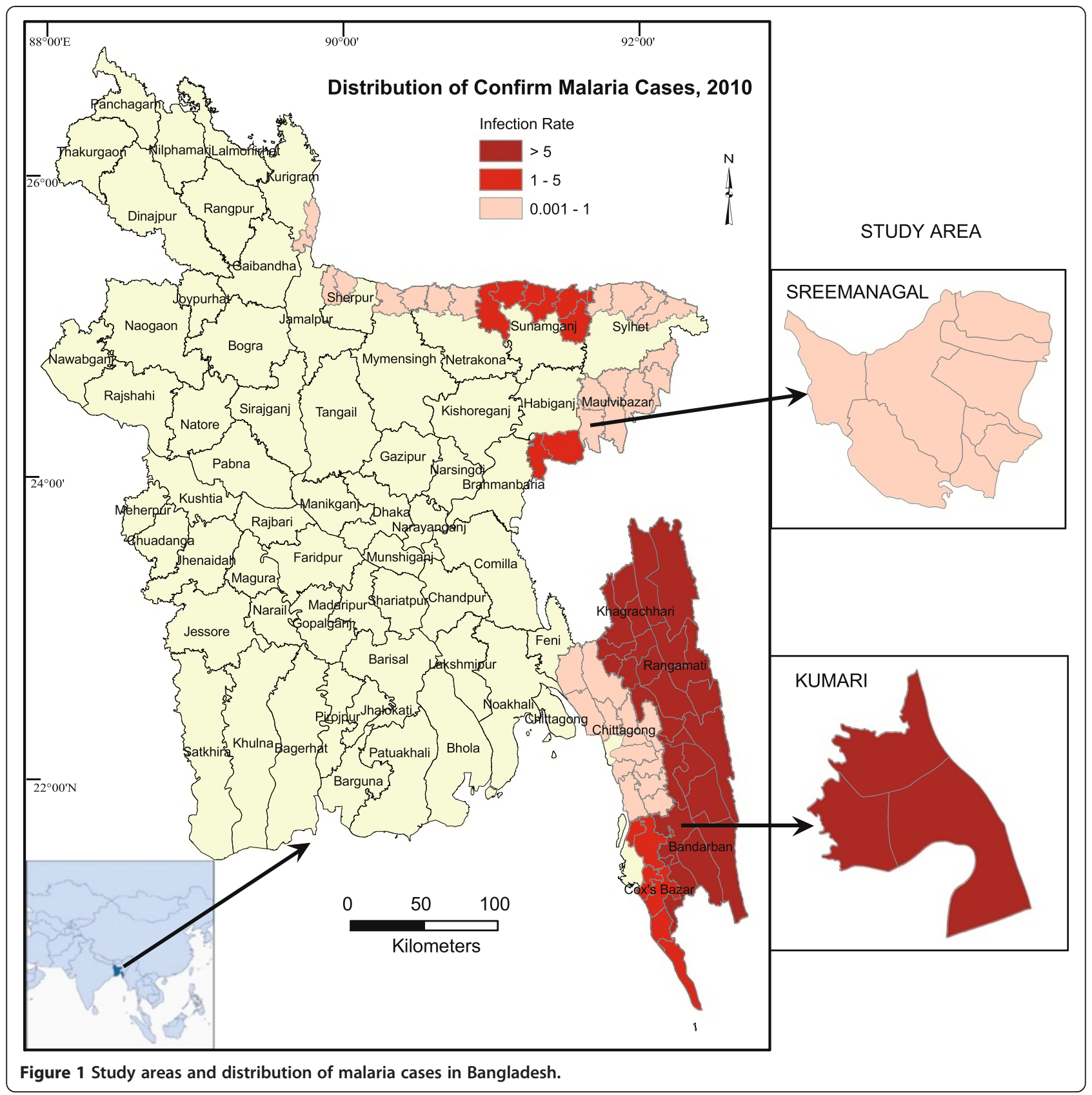

department of Bangladesh. Study areas are located in a tropical monsoon climate characterized by marked seasonal variations. Abundant rainfall (monthly average $595.12 \mathrm{~mm}$ ) during the monsoon (July-October) is followed by a cool winter period (November-February), then a hot, dry summer (March-June). The winter season is dry and accounts for $2-4 \%$ (average $1.88 \mathrm{~mm}$ ) of the annual rain. As the winter season progress into the summer hot season, rainfall increase $(10-35 \%$ of the total annual rain). In the hot summer season, the average maximum temperature is $34^{\circ} \mathrm{C}$ and the minimum is $21^{\circ} \mathrm{C}$. Average maximum temperature in winter is $29^{\circ} \mathrm{C}$ and the minimum is $11^{\circ} \mathrm{C}$. The average monthly relative humidity for the whole year ranges from 63.13 to $87.73 \%$. The relative humidity is over $80 \%$ during June to September. March and April are the least humid months.

\section{Data analysis}

In order to compare counts of female anophelines captured in different periods, we determined the relative density of every mosquito species according to the formula of Kocatas [26] and Simsek [27], in which RD (relative 
density) = NA (number of all specimens of each species collected during each period)/ $\mathrm{N}$ (the number of specimens of all species collected during each period) $\times 100$. Statistical analyses were done on abundance data of the predominant species captured during the study to find out which environmental variables were leading on species distributions in the study areas. Pearson correlation coefficients were computed for the dominant species using SPSS ${ }^{\circ} 16.0$ (๑) SPSS Inc., Chicago, IL. 2007) to study the correlations between mosquito abundance and environmental variables. It was also calculated to know relation between malaria morbidity and environmental variables. Pearson correlation analysis between malaria morbidity and environmental variables were not done with the data of Sreemangal area because morbidity was not recorded in this area during the study period. Canonical correspondence analyses (CCA) [28] have been done using CANOCO 4 for Windows [29] to further explore the associations between species abundance with meteorological variables. It is a multivariate direct gradient analysis technique, where species abundance and composition is directly related to a set of meteorological variables. CCA was carried out in our study because meteorological variables may be highly correlated with one another. For example, rainfall, temperature and humidity are usually very tightly correlated. If so, any one of these variables could be used as a proxy for all others. Generally, it is best to choose the variable which is most likely to be the direct cause of species response, and/or a variable which has been used in other ecological studies. It might not be known beforehand which variables are correlated with each other. In this case, a detailed examination of the correlation matrix would be helpful. A relatively sophisticated way to do this would be to perform CCA. Moreover, it performs well with skewed species distributions, high noise levels, and complex sampling designs [30]. Traditional analyses were used to calculate the minimum infection rate (MIR) of CSP and to estimate the population infection rate from pooled samples. When a pool was positive, only one individual in that pool was considered to be positive.

\section{Results}

\section{Species composition and density}

A total of 2,443 female anophelines, representing 22 species were collected in two selected areas during January 2011 to February 2012 (Table 1). Among the 22 species collected, the most prevalent species was An. vagus ( $\mathrm{n}=1263,51.70 \%)$ followed by An. philippinensis $(\mathrm{n}=560,22.92 \%)$, An. jeyporiensis $(\mathrm{n}=143,5.85 \%)$, An . karwari $(\mathrm{n}=108,4.42 \%)$ and An. peditaeniatus $(\mathrm{n}=97$, $3.97 \%)$ respectively. Few number of samples $(\mathrm{n}<10)$ was collected in the following 10 species; An. aitkenii, An. baimaii, An. nigerrimus, An. nivipes, An. pallidus, An. pseudojamesi, An. tessellatus, An. maculatus, An. hyrcanus group, and An. willmori (Table 1).

\section{Species composition in Kumari}

In total, 2415 female Anopheles mosquito belonging to 22 species were captured in Kumari using LTs ( $\mathrm{n}=$ $2237)$ and PS $(n=178)$. All 22 species were collected using LTs set indoors $(\mathrm{n}=1163)$, and 20 species were collected with outdoor LTs $(\mathrm{n}=1074)$. In contrast, only eight species were collected using PS $(\mathrm{n}=178)$. Anopheles vagus was captured dominant in both LTs and PS in indoor. Relatively high number of An. karwari $(\mathrm{n}=76)$ and An. peditaeniatus $(\mathrm{n}=57)$ were captured in outdoor (Table 1).

\section{Species composition in Sreemangal}

Small number $(\mathrm{n}=28)$ of female anophelines were captured using light trap set both indoors and outdoors during one year sampling period in Sreemangal. Only six species; $A n$. peditaeniatus, An. jeyporiensis, An. annularis, An. karwari, An. philippinensis, and An. vagus were collected from this area. Anopheles peditaeniatus $(\mathrm{n}=15)$ was the dominant species followed by $A n$. jeyporiensis, and An. annularis. Anopheles vagus and An. karwari were captured only in indoors (Table 1).

\section{Gonotrophic stages}

Gonotrophic stages of mosquito were visually classified as unfed (UF), Blood-fed (BF), semi-gravid (SG), or gravid (G). The highest percentage of specimens was UF $(\mathrm{n}=1601,65.53 \%)$, followed by BF $(\mathrm{n}=697,28.53 \%), \mathrm{G}$ $(\mathrm{n}=91,3.72 \%)$, and SG $(\mathrm{n}=54,2.21 \%)$.

\section{Circumsporozoite positive (CSP) rates}

Every female Anopheles were tested for P. falciparum, $P$. vivax 210 and $P$. vivax 247 CSP, of which 10 species were found positive. The CSP positive species were An. annularis, An. baimaii, An. barbirostris Van der Wulp, An. jeyporiensis, An. karwari, An. minimus s.l., An. philippinensis, An. umbrosus, An. vagus and An. wilmori. A total of $22(0.91 \%)$ mosquitoes belonging to eight species were found positive for $P$. falciparum, $10(0.41 \%)$ mosquitoes belonging to five species were positive for $P v-210$ and one $(0.04 \%)$ mosquitoes belonging to one species were positive for $P v$-247. Mixed infections were found in 5 females $(0.20 \%)$ anophelines. In total of $P$. falciparum and $P$. vivax infections, the highest infection rate was observed in An. baimaii (2/3, 66.66\%) followed by An. wilmori (2/8, 25\%), An. barbirostris (1/10, 10\%), An. annularis (1/13, 7.69\%), An. umbrosus (1/16, 6.25), An. karwari (3/132, 2.27\%), An. vagus (21/1251, $1.68 \%)$, An. minimus s.l. (1/64, 1.56\%), An. jeyporiensis (1/98, 1.02\%), and An. philippinensis (5/554, 0.90\%) (Table 2). No Anopheles was found CSP positive, collected from Sreemangal, probably because of the low sample size $(\mathrm{n}=28)$. 
Table 1 Female anophelines collected in indoor and outdoor using light traps and pyrethrum spry (PS) from Kumari (Jan-Dec'11), Bandarban and Sreemangal (Feb-Dec'11 \& Jan'12), Moulvibazar, Bangladesh

\begin{tabular}{|c|c|c|c|c|c|c|c|c|c|c|}
\hline \multirow[t]{3}{*}{ Species } & \multicolumn{5}{|l|}{ Kumari } & \multicolumn{3}{|c|}{ Sreemangal } & \multicolumn{2}{|c|}{ Grand Total } \\
\hline & \multicolumn{3}{|c|}{ Light trap } & \multirow{2}{*}{$\begin{array}{l}\text { PS } \\
\text { Indoor }\end{array}$} & \multirow[t]{2}{*}{ Total } & \multicolumn{2}{|c|}{ Light trap } & \multirow[t]{2}{*}{ Total } & \multirow[t]{2}{*}{$\mathbf{N}$} & \multirow[t]{2}{*}{$\mathrm{RD}^{1}$} \\
\hline & Indoor & Outdoor & Total & & & Indoor & Outdoor & & & \\
\hline Anopheles aitkenii & 1 & & 1 & & 1 & & & & 1 & 0.04 \\
\hline An. annularis & 3 & 7 & 10 & 1 & 11 & 3 & 1 & 4 & 15 & 0.61 \\
\hline An. baimaii & 2 & 1 & 3 & & 3 & & & & 3 & 0.12 \\
\hline An. barbirostris & 7 & 3 & 10 & & 10 & & & & 10 & 0.41 \\
\hline An. hyrcanus group & 4 & 2 & 6 & & 6 & & & & 6 & 0.25 \\
\hline An. jamesii & 35 & 20 & 55 & 2 & 57 & & & & 57 & 2.33 \\
\hline An. jeyporiensis & 62 & 71 & 133 & 5 & 138 & 3 & 2 & 5 & 143 & 5.85 \\
\hline An. karwari & 30 & 76 & 106 & & 106 & 2 & & 2 & 108 & 4.42 \\
\hline An. kochi & 16 & 17 & 33 & & 33 & & & & 33 & 1.35 \\
\hline An. maculatus group & 2 & 4 & 6 & & 6 & & & & 6 & 0.25 \\
\hline An. minimus s.l. & 26 & 19 & 45 & 1 & 46 & & & & 46 & 1.88 \\
\hline An. nigerrimus & 2 & 1 & 3 & & 3 & & & & 3 & 0.12 \\
\hline An. nivipes & 2 & 2 & 4 & & 4 & & & & 4 & 0.16 \\
\hline An. pallidus & 4 & & 4 & & 4 & & & & 4 & 0.16 \\
\hline An. peditaeniatus & 24 & 57 & 81 & 1 & 82 & 1 & 14 & 15 & 97 & 3.97 \\
\hline An. pseudojamesi & 3 & 1 & 4 & & 4 & & & & 4 & 0.16 \\
\hline An. philippinensis & 268 & 289 & 557 & 2 & 559 & & 1 & 1 & 560 & 22.92 \\
\hline An. subpictus & 14 & 36 & 50 & 1 & 51 & & & & 51 & 2.09 \\
\hline An. tessellatus & 1 & 4 & 5 & & 5 & & & & 5 & 0.20 \\
\hline An. umbrossus & 9 & 7 & 16 & & 16 & & & & 16 & 0.65 \\
\hline An. vagus & 643 & 454 & 1097 & 165 & 1262 & 1 & & 1 & 1263 & 51.70 \\
\hline An. willmori & 5 & 3 & 8 & & 8 & & & & 8 & 0.33 \\
\hline Grand Total & 1163 & 1074 & 2237 & 178 & 2415 & 10 & 18 & 28 & 2443 & 100.00 \\
\hline
\end{tabular}

${ }^{1}$ Relative density.

\section{Seasonal prevalence in Kumari}

The seasonal distributions of the most abundant species reveal population fluctuations in different months. Anopheles vagus and $A n$. philippinensis were the dominant species present almost throughout the year with major peaks in March and smaller peaks in September but $A n$. baimaii and An. willmori were found during monsoon (July -September) only. Although the population of An. karwari decreased greatly in May and December, it was collected in small numbers throughout the sampling period with peaks in February. Highest and lowest densities of anophelines were found in March and December respectively in Kumari (Figure 2).

\section{Seasonal prevalence in Sreemangal}

Mosquito density was reached peak during April in Sreemangal. The density of An. minimus s.l. was higher during January to March. Abundance of An. annularis followed the same general trend as that for An. minimus s.l. but with smaller numbers.
Association with climatic variables and morbidity

Bivariate Pearson's correlation analyses of most abundant species with climatic variables showed that the abundance of An. baimaii $(p<0.01)$ and An. willmori $(p<0.01)$ has strong positive correlation with lag rainfall in Kumari. But we did not find any significant relation with rainfall and most other Anopheles species in Kumari as well as in Sreemangal. The effect of temperature were not found as an important variable on the abundance of anophelines mosquitoes in both the study areas except An. karwari $(\mathrm{R}=-0.58, p<0.05)$. Strong negative correlation between relative humidity and abundance of $A n$. karwari $(p<0.01)$, An. minimus s.l. $(p<0.01)$, An. annularis $(p<0.01)$, and $A n$. jeyporiensis $(p<0.01)$ were observed in our study. We found significant relation between An. willmori $(p<0.05)$ and malaria morbidity when Spearman's correlation analyses was done. Insignificant impact of 1 month lag rainfall and temperature on the malaria morbidity were found in Kumari. Highest number of malaria cases was recorded during July but rainfall was higher 
Table 2 Species and number of Anopheles tested and their CSP positive rates

\begin{tabular}{|c|c|c|c|c|c|c|c|}
\hline \multirow{2}{*}{$\begin{array}{l}\text { Anopheles } \\
\text { species }\end{array}$} & \multirow{2}{*}{$\begin{array}{l}\text { Number } \\
\text { tested }\end{array}$} & \multicolumn{5}{|c|}{ CSP positive } & \multirow{2}{*}{$\begin{array}{l}\text { Infection } \\
\text { rate (\%) }\end{array}$} \\
\hline & & $\mathrm{Pf}$ & Pv210 & Pv247 & Mixed (Pf, Pv210) & Total & \\
\hline aitkenii & 2 & - & - & - & - & 0 & 0.00 \\
\hline annularis & 13 & 1 & - & - & - & 1 & 7.69 \\
\hline baimaii & 3 & 2 & - & - & - & 2 & 66.67 \\
\hline barbirostris & 10 & 1 & - & - & - & 1 & 10.00 \\
\hline hyrcanus & 6 & - & - & - & - & 0 & 0.00 \\
\hline jamesii & 57 & - & - & - & - & 0 & 0.00 \\
\hline jeyporiensis & 98 & - & - & 1 & - & 1 & 1.02 \\
\hline karwari & 132 & 1 & 2 & - & - & 3 & 2.27 \\
\hline kochi & 36 & - & - & - & - & 0 & 0.00 \\
\hline maculatus & 7 & - & - & - & - & 0 & 0.00 \\
\hline minimus s.l. & 64 & - & 1 & - & - & 1 & 1.56 \\
\hline nigerrimus & 3 & - & - & - & - & 0 & 0.00 \\
\hline nivipes & 4 & - & - & - & - & 0 & 0.00 \\
\hline pallidus & 8 & - & - & - & - & 0 & 0.00 \\
\hline peditaeniatus & 82 & - & - & - & - & 0 & 0.00 \\
\hline pheudojamesi & 4 & - & - & - & - & 0 & 0.00 \\
\hline philippinensis & 554 & 1 & 3 & - & 1 & 5 & 0.90 \\
\hline splendidus & 1 & - & - & - & - & 0 & 0.00 \\
\hline subpictus & 51 & - & - & - & - & 0 & 0.00 \\
\hline tessellatus & 5 & - & - & - & - & 0 & 0.00 \\
\hline umbrosus & 16 & 1 & - & - & - & 1 & 6.25 \\
\hline vagus & 1251 & 14 & 3 & - & 4 & 21 & 1.68 \\
\hline willmori & 8 & 1 & 1 & - & - & 2 & 25.00 \\
\hline Total & 2415 & 22 & 10 & 1 & 5 & 38 & 1.57 \\
\hline
\end{tabular}

during August (Figure 3). Significant positive correlation were observed between malaria cases and relative humidity $(p<0.05)$ in Kumari (Table 3).

CCA was performed to further explore the associations between species abundance with meteorological variables. The ordination diagram produced by CCA (Figure $4 \& 5$ ) shows the relationships between species abundance and meteorological variables in Kumari and Sreemangal. The triangles represent species abundance and the arrows are meteorological variables. The direction and length of the arrow indicates the association of the variable and how it correlates with the species composition axes. The angle between arrows shows correlations among the climatic variables. The position of the triangles (species) exposes the environmental preferences of each species. Lag rainfall and relative humidity were the most significant variables influencing An. baimaii, An. willmori, An. vagus, and An. subpictus density in Kumari. Abundance of these four species was positively related to malaria cases. The abundance of other species was negatively associated with environmental variables (Figure 4). We did not find any positive association with environmental variables and female anophelines abundance in Sreemangal (Figure 5). Canonical Correspondence Analysis (CCA) showed a low species-environment correlation with Eigenvalues $(\lambda)$ of the first, second, third and fourth axes of $0.195,0.107,0.044$, and 0.033 , respectively. The Eigenvalue is a reasonable measure of the strength of an ordination axis. It is actually equal to the (maximized) dispersion of the species scores on the ordination axis, and is thus a measure of importance of the ordination axis. The first ordination axis has the largest Eigenvalue, the second axis is the second largest Eigenvalue, and so on. The Eigenvalues of CCA all lie between 0 and 1 . Values over 0.5 often denote a good separation of the species along axis.

\section{Discussion}

Highest peaks of the female anophelines species density were observed during March and smallest peaks during September in Kumari and there were no positive relation with rainfall, and majority of the anopheline species. We 


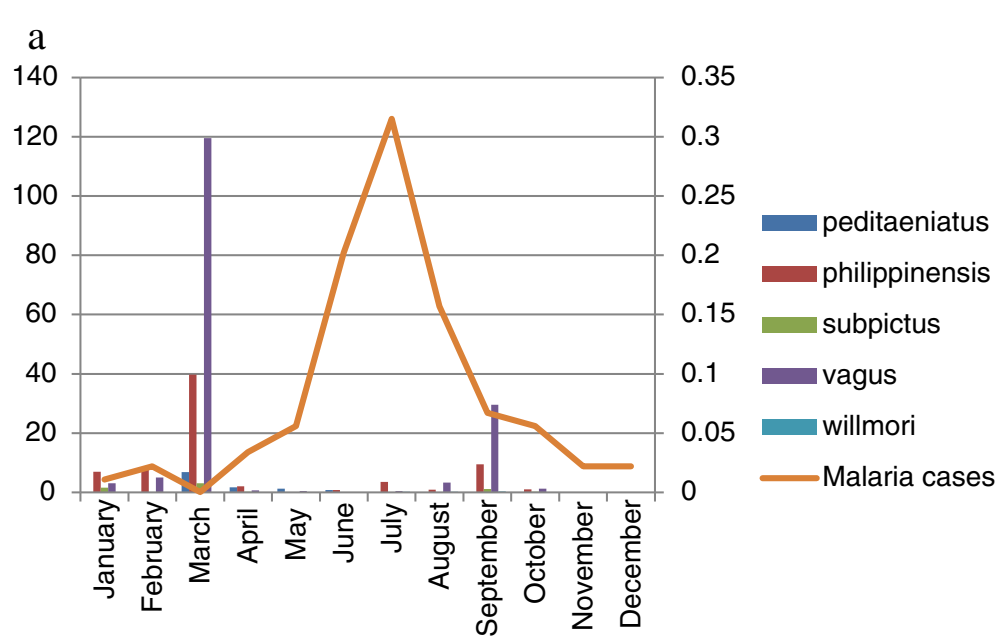

b

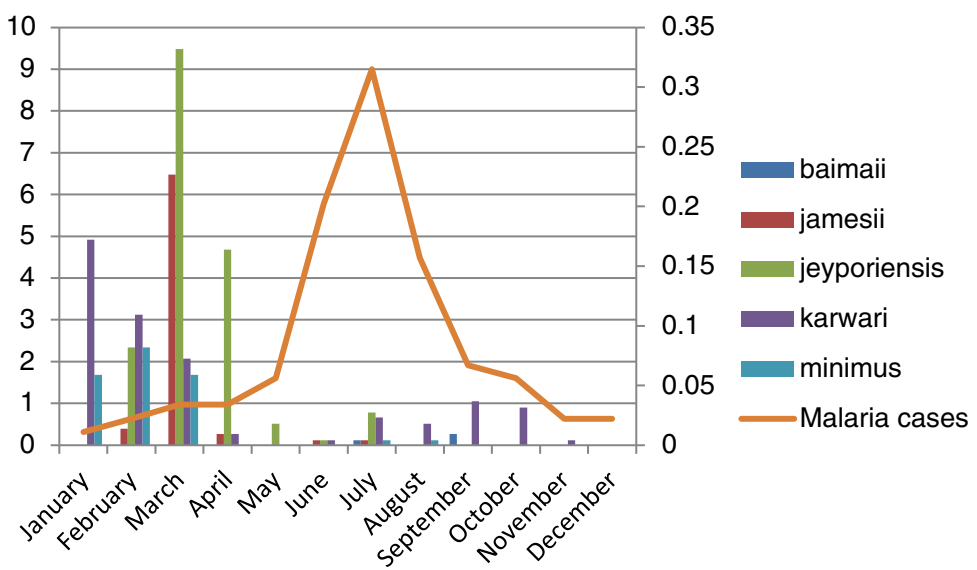

Figure 2 Monthly abundance of anophelines species and malaria morbidity in Kumari. a. Anopheles peditaeniatus, An. philippinensis, An. subpictus, An. vagus, An. willmori and malaria cases. b. Anopheles baimaii, An. jamesii, An. jeyporiensis, An. karwari, An. minimus and malaria cases.

did not found any significant correlation with rain fall, and known malaria vector; An. minimus s.l., An. philippinensis, An. vagus, and $A n$. annularis. It may because of association with rice fields and irrigated cropland, where the females deposit their eggs on moist soil, which was supported by

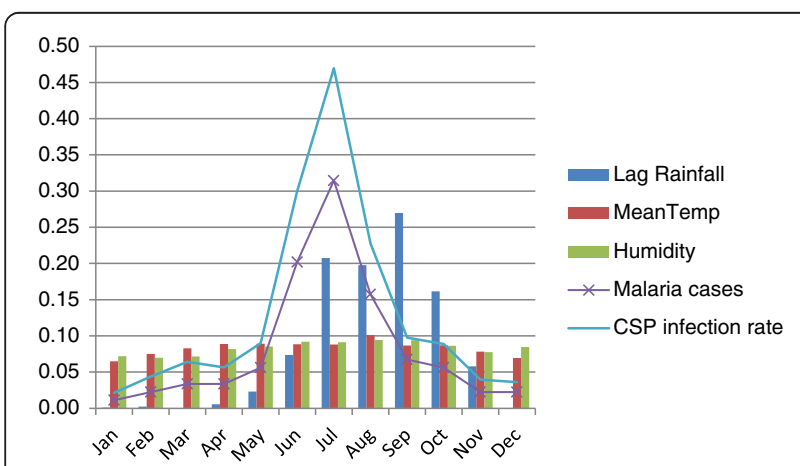

Figure 3 Correlation among CSP infection, malaria morbidity and environmental variables in Kumari.
Meisch [31]. Investigation is needed in these respects in Bangladesh. Like us, Rahman et al. [32] did not found correlation between density and rainfall in Malaysia. The observed results may be because Bangladesh has very high vector species diversity and vectors suited to different breeding habitats. There was mark reduction of mosquito abundance during May to August which increased back in September in our study. The species density reduction during this period may be due to the heavy rainfall, which flushes out breeding sites, larvae, and pupae. It also causes mechanical damage and egg mortality, therefore, reduces the adult abundance [33]. Usually, mosquitoes get optimum $\left(22^{\circ}-30^{\circ} \mathrm{C}\right)$ temperature [34] for rising population after the winter (March and April) in Kumari. The abundance of principle malaria vector; $A n$. baimaii and suspected vector; An. willmori were found strongly associated with rainfall in our study. Anopheles baimaii inhabit forested mountains and foothills, cultivated forests, plantations (e.g. rubber) and forest fringes [35] and An. willmori breeds in slow 
Table 3 Pearson's correlation coofficient (R) of most abundance species with climatic variables and their significant (p) value

\begin{tabular}{|c|c|c|c|c|c|c|c|c|c|}
\hline \multirow[t]{2}{*}{ Place } & \multirow[t]{2}{*}{ Species } & \multicolumn{2}{|c|}{ Lag Rain fall } & \multicolumn{2}{|c|}{ Mean Temperature } & \multicolumn{2}{|c|}{ Relative Humidity } & \multicolumn{2}{|c|}{ Malaria morbidity } \\
\hline & & $\mathbf{R}$ & $p$-value & $\mathbf{R}$ & $p$-value & $\mathbf{R}$ & $p$-value & $\mathbf{R}$ & $p$-value \\
\hline \multirow[t]{8}{*}{ Kumari } & An. baimaii & 0.737 & $0.006^{*}$ & 0.175 & 0.587 & 0.485 & 0.110 & 0.312 & 0.323 \\
\hline & An. jeyporiensis & -0.409 & 0.187 & 0.26 & 0.935 & -0.503 & 0.096 & -0.224 & 0.484 \\
\hline & An. karwari & -0.275 & 0.388 & -0.582 & $0.047^{*}$ & -0.664 & $0.019^{*}$ & -0.325 & 0.303 \\
\hline & An. minimus s.l. & -0.467 & 0.126 & -0.511 & 0.089 & -0.804 & $0.002^{*}$ & -0.347 & 0.270 \\
\hline & An. peditaeniatus & -0.368 & 0.239 & 0.078 & 0.809 & -0.383 & 0.219 & -0.181 & 0.574 \\
\hline & An. philippinensis & -0.192 & 0.549 & -0.107 & 0.740 & -0.466 & 0.127 & -0.199 & 0.535 \\
\hline & An. vagus & -0.125 & 0.700 & 0.005 & 0.988 & -0.338 & 0.282 & -0.188 & 0.559 \\
\hline & An. willmori & 0.957 & $0.00^{*}$ & 0.510 & 0.09 & 0.694 & $0.012^{*}$ & 0.486 & 0.110 \\
\hline \multirow[t]{3}{*}{ Sreemangal } & An. annularis & -0.332 & 0.292 & -0.156 & 0.628 & -0.734 & $0.007^{*}$ & - & - \\
\hline & An. jeyporiensis & -0.295 & 0.352 & 0.082 & 0.801 & -0.747 & $0.005^{*}$ & - & - \\
\hline & An. peditaeniatus & -0.171 & 0.594 & 0.127 & 0.694 & -0.446 & 0.146 & - & - \\
\hline
\end{tabular}

running stream margins, rice fields, pits and wells [36], seems to be positively associated with rainfall [37]. Olson and Meek [38] and Focks et al. [39] reported that soil moisture is a major factor affecting the abundance of some species. However, measurements of soil moisture were not included in this study.

We did not found any significant association with temperature; mosquito density and malaria incidence in our study. Certainly, temperature is directly affecting mosquito breeding, survival, and behavior and also malaria transmission [40-43]. We were unable to detect a significant relationship with this factor, because the temperature ranges in this region are always suitable for mosquito breeding and development. Moreover, statistical significance alone does not always unclouded the complex biological dynamics of mosquito and temperature.

Though, rainfall is the major key factor to enhance the malaria transmission in several countries [44-46]. However, it was negatively correlated with malaria cases in India [47]. We found moderate relation $(R=0.573, P=0.052)$ between the number of malaria cases and rainfall in Bangladesh which was supported by Gupta [48]. Haque et al. [2] investigated the relationship between climatic parameters and malaria cases over the last 20 years in the malaria endemic district of Chittagong hill tracts of Bangladesh and showed insignificant relation. But, Wiwanitkit [49] reported that

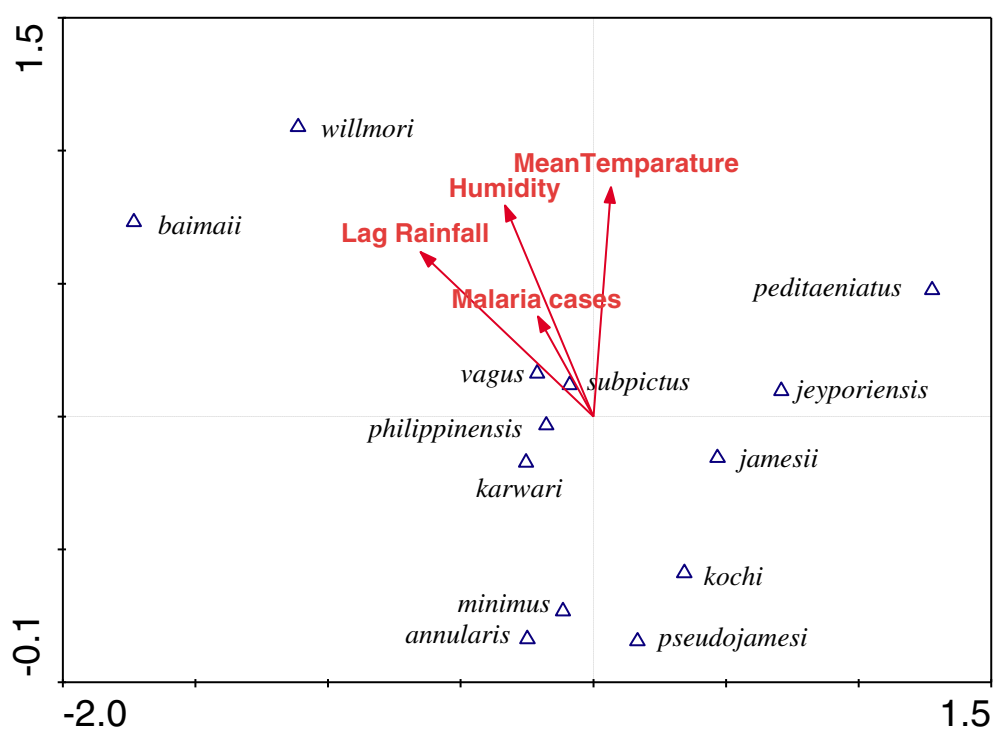

Figure 4 Ordination diagram of most abundance anophelines species and environmental variables of Kumari, Bandarban, produced from canonical correspondence analysis (CCA). 


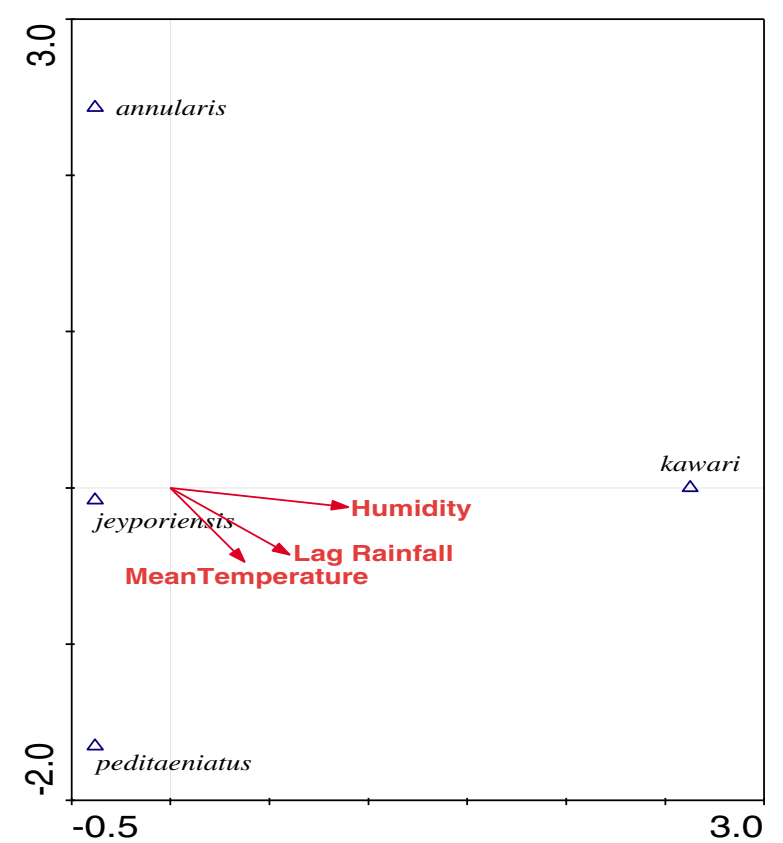

Figure 5 Ordination diagram of most abundance anophelines species and environmental variables of Sreemangal, produced from canonical correspondence analysis (CCA).

malaria cases were positively associated with rainfall in Thailand. Briet et al. [50] showed that malaria cases increased with lower rainfall and that the region with the highest rainfall had the least malaria. Malaria incidence and relative humidity were positively associated when not considering the effect of multiple factors. However, no association was observed in our study when computed combined effect of multiple factors. Bhattacharya et al. [47] accounted humidity levels between 55 and 80\% were appropriate for both $P$. falciparum and $P$. vivax and this range of humidity are present throughout the year in Bangladesh. It was also reported that the malaria risk at $80 \%$ humidity was double as that of $60 \%[42,51]$.

Based on the observation of Anopheles monthly distribution and malaria prevalence, An. baimaii, An. willmori, An. vagus, and $A n$. subpictus seem to potential vector of malaria in the study area. This hypothesis was supported by their anthropophilic and opportunistic feeding behavior [52] and Plasmodium infection rate $[1,9]$.

\section{Conclusions}

The study demonstrates that the incidences of malaria all year round in Kumari, Bangladesh, due to the favorable environmental conditions. Therefore, integrated vector management system covering year round should be adopted to reduce of malaria morbidity and mortality in Bangladesh. Additionally, basic and applied research on the ecological, social and economic determinants of the disease is required to promote the regular assessment of a country's malaria situation. Like other studies, we did not find significant relationships of rainfall and temperature with all anophelines; even so, this may be due to the fact that other studies used diverse methodologies in different regions of the globe, where the vector ecology is pretty different. Multiple environmental factors are responsible for mosquito breeding and malaria infection in the Bangladesh. This study recommends vector ecologists to cautiously consider the complex nature of the relationship between malaria vectors and climate variables. Detailed studies of vector bionomics, continuous monitoring and malaria transmission dynamics is essential for predicting outbreaks of disease and, if necessary, control of pest mosquitoes in Bangladesh.

\section{Competing interests}

The authors declare that they have no competing interests.

\section{Authors' contributions}

$\mathrm{TN}$ and $\mathrm{KB}$ designed the study. $\mathrm{KB}$, conducted the fieldwork. KB performed the ELISA and PCR assay of KB and TN collaborated in writing the manuscript. Both authors read and approved the final manuscript.

\section{Acknowledgements}

We thank the villagers' for their permission to collect mosquitoes in their houses. In particular, we thank the community leaders. We are grateful to Bangladesh meteorological department for providing environmental data. We are also grateful to malaria control program Bangladesh and local hospitals for providing malaria morbidity data. We thank MS Shahen for help during fieldwork. Special thanks go to Kh. Hasan Mahmud, Assistant Professor, Department of Geography and Environment, JU and Morshed Alam, Lecturer, Department of Statistics, JU for valuable suggestions and help with data analysis. Grateful thanks to Dr. Robbin Lindasy and NP Maheswary for critical review of our manuscript. We acknowledge the Laboratory of Ecology of Kanazawa University, Japan, for providing technical and laboratory space for the study.

\section{Author details}

'Laboratory of Entomology, Department of Zoology, Jahangirnagar University, Savar, Dhaka 1342, Bangladesh. 'Laboratory of Ecology, Faculty of Natural Science and Technology, Kanazawa University, Kanazawa, Japan.

Received: 15 April 2014 Accepted: 26 August 2014

Published: 18 September 2014

\section{References}

1. Alam MS, Khan MGM, Chaudhury N, Deloer S, Nazib F, Bangali AM, Haque R: Prevalence of anopheline species and their Plasmodium infection status in epidemic-prone border areas of Bangladesh. Malar J 2010, 9:15.

2. Haque U, Hashizume M, Glass GE, Dewan AM, Overgaard HJ, Yamamoto T: The Role of Climate Variability in the Spread of Malaria in Bangladeshi Highlands. PLOS One 2010, 5(12):e14341. doi:10.1371/journal.pone.0014341.

3. Khan $A Q$, Talibi SA: Epidemiological assessment of malaria transmission in an endemic area of East Pakistan \& significance of congenital immunity. WHO Bull 1972, 46:783-792.

4. Elias M, Dewan R, Ahmed R: Vectors of malaria in Bangladesh. J Prev Social Med 1982, 1:20-28.

5. Maheswary NP, Habib MA, Elias M: Incrimination of Anopheles aconitus Donitz as a vector of epidemic malaria in Bangladesh. SE Asian J Trop Med 1992, 23:798-801.

6. Maheswary NP, Khan Z, Molla FR, Haq Ml: Incrimination of Anopheles annularis van der Wulp-1854 as an epidemic malaria vector in Bangladesh. SE Asian J Trop Med 1993, 24:776-778.

7. Maheswary NP, Majumdar S, Chowdhury AR, Faruque MS, Montanari RM: Incrimination of Anopheles vagus Donitz, 1902 as an epidemic malaria vector in Bangladesh. Indian J Malariol 1994, 31:35-38. 
8. M\&PDC: Mosquito vectors in Bangladesh survey report 2008-2009. In Malaria and Parasitic Diseases Control, Directorate General of Health in Bangladesh; 2009:123.

9. Bashar K, Tuno N, Ahmed TU, Howlader AJ: False positive of circumsporozoite protein (CSP)-ELISA in zoophilic anophelines in Bangladesh. Acta Trop 2012, 125:220-225.

10. Tandon N, Hati AK: Some recent malaria problems in the hill district of eastern India. Memoirs School Entomol 1978, 6:117-212.

11. Wegbreit J, Reisen WK: Relationships among weather, mosquito abundance, and encephalitis virus activity in California: Kern County 1990-98. J Am Mosa Contr Assoc 2000, 16:22-27.

12. Camargo LM, Ferreira MU, Krieger H, Camargo EP, Pereira Da Silva L: Unstable hypoendemic malaria in Rondonia (Western Amazon Region, Brazil): epidemic outbreaks and work associated incidence in an agro-industrial rural settlement. Am J Trop Med Hyg 1994, 51:16-25.

13. Rubio-Palis $Y$, Zimmerman RH: Ecoregional classification of malaria vectors in the neotropics. J Med Entomol 1997, 34:499-510.

14. Conn JE, Wilkerson RC, Segura MNO, Souza RTL, Schlichting CD, Wirtz RA, Póvoa MM: Emergence of a new Neotropical malaria vector facilitated by human migration and changes in land use. Am J Trop Med Hyg 2002, 66:18-22.

15. Vittor AY, Gilman RH, Tielsch J, Glass G, Shields T, Lozano WS, Pinedo-Cancino V, Patz JA: The effect of deforestation on the human-biting rate of Anopheles darlingi, the primary vector of falciparum malaria in the Peruvian Amazon. Am J Trop Med Hyg 2006, 74:3-11.

16. Rosa-Freitas MG, Tsouris P, Peterson AT, Hon'orio NA, Barros FS, de Aguiar DB, GurgelHda C, de Arruda ME, Vasconcelos SD, Luitgards-Moura JF: An ecoregional classification for the state of Roraima, Brazil: the importance of landscape in malaria biology. Mem Inst Oswaldo Cruz 2007, 102(3):349-357.

17. Alten B, Bellini R, Caglar SS, Simsek FM, Kaynas S: Species composition and seasonal dynamics of mosquitoes in the Belek region of Turkey. J Vector Ecol 2000, 25:146-154.

18. WHO: Manual on practical entomology in malaria. Part II: Methods and techniques. In Division of malaria and other parasitic diseases; 1975.

19. Christophers SR: The fauna of British India, including Ceylon and Burma. Diptera 4, Family Culicidae. In Tribe Anopheline. London: Taylor \& Francis; 1933:371.

20. Puri IM: Synoptic table for the identification of the anopheline mosquitoes of India. In Health Bull No 10. 5th edition. India: Malaria Bureau, Govt India Press; 1960.

21. Harrison BA, Scanlon JE: Medical entomology studies-II. The subgenus Anopheles in Thailand. Contrib Am Entomol Inst 1975, 12:1-307.

22. M\&PDC: Malaria Vectors and Other Anopheles Mosquitoes in Bangladesh. In Entomology Division, Malaria and Parasitic Diseases Control, Directorate General of Health in Bangladesh; 2009:22.

23. Burkot TR, Williams JL, Schneider L: Identification of Plasmodium falciparum infected mosquitoes by a double antibody enzyme-linked immunosorbent assay. Am J Trop Med Hyg 1984, 33:783-788.

24. Wirtz RA, Charoenvit Y, Burkot TR, Esser KM, Beaudoin RL, Collins WE, Andre RG Evaluation of monoclonal antibodies against Plasmodium vivax for ELISA development. Med Vet Entomol 1991, 5:17-22.

25. Durnez L, Bortel WV, Denis L, Roelants $P$, Veracx A, Trung HD, Sochantha T, Coosemans M: False positive circumsporozoite protein ELISA: a challenge for the estimation of the entomological inoculation rate of malaria and for vector incrimination. Malar J 2011, 10:195

26. Kocatas A: EkolojiveÇevreBiyolojisi. Izmir: EgeÜnivMatbaasi; 1992:564s.

27. Simsek FM: Seasonal frequency and relative density of larval populations of mosquito species (Diptera: Culicidae) in Anhurfa Province, Turkey. Turk J Zool 2006, 30:383-392.

28. TerBraak CJF: Canonical correspondence analysis: A new eigenvector technique for multivariate direct gradient analysis. Ecology 1986, 67:1167-1179.

29. TerBraak CJF, Smilauer P: CANOCO reference manual and user's quide to Canoco for Windows: software for canonical community ordination (version 4). New York: Microcomputer Power; 1998.

30. Palmer MW: Putting things in even better order: The advantages of canonical correspondence analysis. Ecology 1993, 74:2215-2230.

31. Meisch MV: The dark rice field mosquito Psorophora columbiae. Wing Beats 1994, 5:8.

32. Rahman WA, Abu-Hasan A, Adanan CR: Seasonality of Anopheles aconitus mosquitoes, a secondary vector of malaria, in an endemic village near the Malaysia-Thailand border. Acta Trop 1993, 55:263-265.
33. Amusan AAS, Mafiana CF, Idowu AB, Oke OA: A survey of adult mosquitoes in the hostels of the University of Agriculture, Abeokuta, Ogun State, Nigeria. Nigeria J Parasitol 2003, 24:167-172.

34. Pratt HD, Moor CG: Mosquitoes of Public Health Importance and their Control. Atlanta: US department of health and human service, CDC; $1993: 55$ p.

35. Rosenberg R: Forest malaria in Bangladesh. III. Breeding habits of Anopheles dirus. Am J Trop Med Hyg 1982, 31:192-201.

36. Rattanarithikul R, Green CA, Panyim S, Noigamol C, Chanaimongkol S, Mahapibul P: Larval habitats of malaria vectors and other Anopheles mosquitoes around a transmission focus in northwestern Thailand. J Am Mosq Control Assoc 1995, 11:428-433.

37. Obsomer V, Defourny P, Coosemans M: The Anopheles dirus complex: spatial distribution and environmental drivers. Malar J 2007, 6:26.

38. Olson JK, Meek CL: Soil moisture conditions that are most attractive to ovipositing females of Psorophora columbiae in Texas rice lands. Mosq News 1977, 37:19-26.

39. Focks DA, McLaughlin RE, Smith BM: A dynamic life table model of Psorophora columbiae in the southern Louisiana rice agro ecosystem with supporting hydrological sub model. Part I. Analysis of literature and model development. J Am MosqContrAssoc 1988, 4:266-281.

40. Craig MH, Snow RW, le Sueur D: A climate-based distribution model of malaria transmission in sub-Saharan Africa. Parasitol Today 1999, 15(3):105-111

41. Paaijmans KP, Imbahale SS, Thomas MB, Takken W: Relevant microclimate for determining the development rate of malaria mosquitoes and possible implications of climate change. Malaria J 2010, 9:196.

42. Ye' Y, Louis V, Simboro S, Sauerborn R: Effect of meteorological factors on clinical malaria risk among children: an assessment using village-based meteorological stations and community-based parasitological survey. BMC Public Health 2007, 7:101.

43. Bi P, Tong S, Donald K, Parton KA, Ni J: Climatic variables and transmission of malaria: a 12-year data analysis in Shuchen County, China. Public Health Rep 2003, 118:65-71.

44. Thomson MC, Mason SJ, Phindela T, Connor SJ: Use of rainfall and sea surface temperature monitoring for malaria early warning in Botswana. Am J Trop Med Hyg 2005, 73(1):214-221.

45. Thomson MC, Doblas-Reyes FJ, Mason SJ, Hagedorn R, Connor SJ, PhindelaT MAP, Palmer TN: Malaria early warnings based on seasonal climate forecasts from multimodel ensembles. Nature 2006, 439:576-579.

46. Zhou G, Minakawa N, Andrew K, Guiyun Y: Association between climate variability and malaria epidemics in the east African highlands. ProcNat/AcadSci USA 2004, 101:2375-2380.

47. Bhattacharya S, Sharma C, Dhif Thomson man R, Mitra A: Climate change and malaria in India. Current Sci 2006, 90:369-374.

48. Gupta R: Correlation of rainfall with upsurge of malaria in Rajasthan J Assoc Physicians India 1996, 44:385-389.

49. Wiwanitkit $\mathrm{V}$ : Correlation between rainfall and the prevalence of malaria in Thailand. J Infect 2006, 52:227-230

50. Briet J, Vounatsou P, Gunawardena D, Galappaththy N, Amerasinghe P: Temporal correlation between malaria and rainfall in Sri Lanka. Malar J 2008, 7:77.

51. Tian L, Bi Y, Ho SC, Liu W, Liang S, Goggins WB, Chan EY, Zhou S, Sung JJ: One-year delayed effect of fog on malaria transmission: a time-series analysis in the rain forest area of Mengla County, south-west China. Malar J 2008, 7:110.

52. Bashar K, Tuno N, Ahmed TU, Howlader AJ: Blood-feeding patterns of Anopheles mosquitoes in a malaria-endemic area of Bangladesh. Parasit Vectors 2012, 5:39.

doi:10.1186/1756-3305-7-442

Cite this article as: Bashar and Tuno: Seasonal abundance of Anopheles mosquitoes and their association with meteorological factors and malaria incidence in Bangladesh. Parasites \& Vectors 2014 7:442. 